\title{
Case in Point
}

\section{Multisystem Inflammatory Syndrome in Children (MIS-C) Associated With COVID-19/Incomplete Kawasaki Disease}

Natalia Reborido Campoy, MD ${ }^{1} \bullet$ Kanupriya Gulati, DO ${ }^{1}$ Kathleen Morris, MD² $\bullet$ Francisco Delgado, MD³

n December 2019, an outbreak of a new disease (COVID-19) due to a novel coronavirus (SARS-CoV-2) began in China, and the infection quickly spread around the world. ${ }^{1,2}$ Current evidence supports that children (ie, persons younger than 18 years) account for $1 \%$ to $5 \%$ of all diagnosed cases of COVID-19, ${ }^{3}$ and, when infected, they frequently are asymptomatic or experience only mild symptoms. In children younger than 21 years, a significant systemic inflammatory response due to COVID-19 infection is increasingly being reported.

The Centers for Disease Control and Prevention (CDC) defines this syndrome as multisystem inflammatory syndrome in children (MIS-C) associated with COVID-19. ${ }^{4}$ It is also called Kawasaki-like disease or incomplete Kawasaki disease (KD), since some patients partially meet the criteria for $\mathrm{KD}$, and the pathophysiology seems to be related. As of today, no guidelines exist for the treatment of persons with MIS-C, and management is based on the current treatment of $\mathrm{KD}$, which is also supported by case reports.

\section{AFFILIATIONS:}

${ }^{1}$ Department of Internal Medicine, Ascension St. Vincent Hospital, Indianapolis, Indiana

${ }^{2}$ Department of Cardiology, Ascension St. Vincent Hospital, Indianapolis, Indiana ${ }^{3}$ Department of Infectious Diseases, Ascension St. Vincent Hospital, Indianapolis, Indiana

\section{CITATION:}

Reborido Campoy N, Gulati K, Morris K, Delgado F. Multisystem inflammatory syndrome in children (MIS-C) associated with COVID-19/incomplete Kawasaki disease. Consultant. Published online August 12, 2020.

doi:10.25270/con.2020.08.00005

Received July 1, 2020. Accepted July 21, 2020.

\section{DISCLOSURES:}

The authors report no relevant financial relationships.

\section{CORRESPONDENCE:}

Natalia Reborido Campoy, MD, Ascension St. Vincent Hospital Department of Internal Medicine, 3 North, 2001 W 86th St, Indianapolis, IN 46260

(natalia.reborido.campoy@ascension.org)

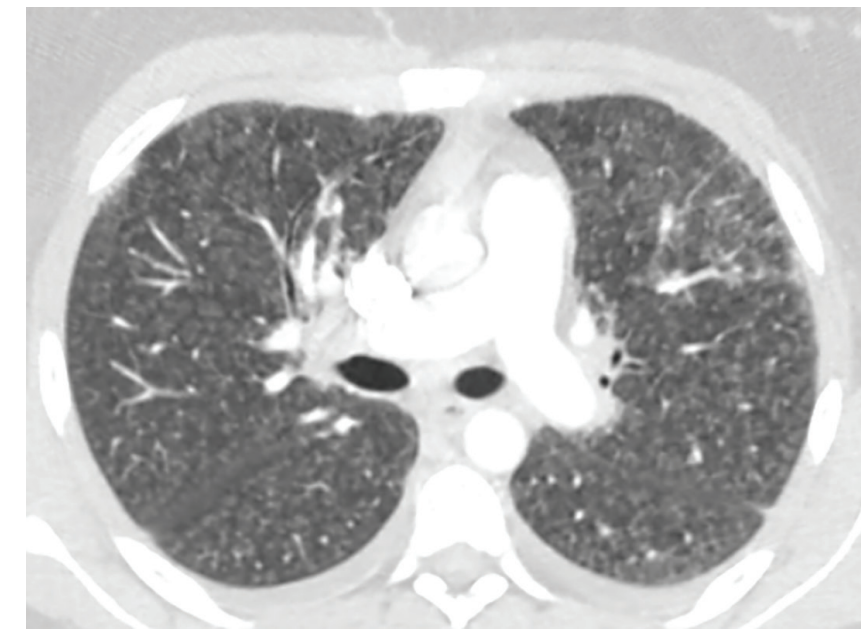

CT scans at the patient's second ED visit showed a significant Increase in interstitial infiltrates with mediastinal and hilar adenopathy and small bilateral pleural effusions, findings concerning for infection.

This case highlights a 20-year-old woman who met the criteria for MIS-C and was treated with intravenous immunoglobulin (IVIG) therapy and aspirin with successful results.

\section{CASE REPORT}

A 20-year-old woman with an unremarkable medical history presented to an outside emergency department (ED) in midMay, 2020, with a 5-day history of gastrointestinal (GI) tract symptoms including nausea, vomiting, and diarrhea, as well as dyspnea with a dry cough. She also reported a transient rash on her arms and inner thighs lasting less than 1 day, which had resolved by the time of presentation to the ED.

At that visit, computed tomography (CT) scans of the abdomen and pelvis were remarkable for some lower-lung opacities. Results of a COVID-19 test were negative. She was briefly 
Table. Case Definition for MIS-C Associated With COVID-19

Age $<21$ y

\section{AND}

Fever $>38.0^{\circ} \mathrm{C}$ for $\geq 24 \mathrm{~h}$, or report of subjective fever lasting $\geq 24 \mathrm{~h}$

\section{AND}

Laboratory markers of inflammation (including but not limited to 1 or more of the following: elevated level of CRP, ESR, fibrinogen, procalcitonin, D-dimer, ferritin, LDH, or IL-6; elevated neutrophil count, reduced lymphocyte count, and low albumin level

\section{AND}

Evidence of clinically severe illness requiring hospitalization, with multisystem $(\geq 2)$ organ involvement (check all applicable below):

- Cardiac (eg, shock, elevated troponin level, elevated BNP, abnormal echocardiography findings, arrhythmia)

- Renal (eg, acute kidney injury or kidney failure)

- Respiratory (eg, pneumonia, ARDS, PE)

- Hematologic (eg, elevated D-dimer level, thrombophilia, thrombocytopenia

- Gastrointestinal (eg, elevated bilirubin, elevated liver enzymes, diarrhea)

- Dermatologic (eg, rash, mucocutaneous lesions)

- Neurologic (eg, CVA, aseptic meningitis, encephalopathy)

\section{AND}

No alternative plausible diagnosis

\section{AND}

Positive for current or recent SARS-CoV-2 infection by (check all applicable below)

- RT-PCR

- Serology

- Antigen test

\section{OR}

COVID-19 exposure within the 4 weeks prior to symptom onset

Abbreviations: ARDS, acute respiratory distress syndrome; BNP, brain-type natriuretic peptide; CRP, C-reactive protein; CVA, cerebrovascular accident; ESR, erythrocyte sedimentation rate; IL-6, interleukin 6; LDH, lactate dehydrogenase; PE, pulmonary embolism; RT-PCR, reverse transcription polymerase chain reaction. hospitalized due to transient decreases in oxygen saturation to as low as $88 \%$, which required supplemental oxygen at $2 \mathrm{~L} / \mathrm{min}$ via a nasal cannula to restore her oxygen saturation to greater than $95 \%$.

Given the patient's symptoms and imaging findings, a diagnosis of pneumonitis/bronchiolitis was made. The next day, given clinical improvement in her condition, the patient was weaned from supplemental oxygen and ultimately was discharged home with a prescription for oral levofloxacin.

Two days after her initial presentation, the patient returned to the same outside ED for worsening dyspnea. At that second visit, she was again found to be hypoxic, with oxygen saturation levels ranging from $85 \%$ to $87 \%$ requiring supplemental oxygen at $3 \mathrm{~L} / \mathrm{min}$ via a nasal cannula. She was admitted again. CT scans of the chest now showed a significant increase in interstitial infiltrates and some hilar and mediastinal lymphadenopathy, along with small diffuse bilateral interstitial opacities representing pleural effusions (Figure).

She was swabbed again for SARS-CoV-2 twice, 48 hours apart, and results of both RNA polymerase chain reaction (PCR) tests returned negative. Results of tests for Legionella pneumophila, Streptococcus pneumoniae, and HIV, as well as the results of a respiratory viral panel, were negative. Intravenous meropenem had been started empirically while test results were pending. Subsequently, a qualitative SARS-CoV-2 immunoglobulin G $(\operatorname{IgG})$ antibody test was performed, the results of which returned positive. The patient became increasingly hypoxic, with oxygen saturation levels as low as $86 \%$ requiring increases in oxygen supplementation up to $5 \mathrm{~L} / \mathrm{min}$ via nasal cannula to keep the oxygen saturation level above $92 \%$. She was then transferred to our hospital on May 20, 2020, for consideration of enrollment in current COVID-19 convalescent plasma and remdesivir clinical trials.

Upon arrival to the medical floor, the patient's GI tract symptoms had resolved, but hypoxia and dyspnea persisted. She remained tachycardic, with her heart rate in the 130s, and continued to have low-grade fevers of $38^{\circ} \mathrm{C}$, but she was hemodynamically stable.

Admission laboratory test results included the following values: hemoglobin, $10.8 \mathrm{~g} / \mathrm{dL}$; mean corpuscular volume, $89 \mu^{3}$; white blood cell count, $9100 / \mu \mathrm{L}$ with lymphocytopenia (absolute lymphocyte count, $510 / \mu \mathrm{L}$ ); fibrinogen, $716 \mathrm{mg} / \mathrm{dL}$; D-dimer, $3.26 \mu \mathrm{g} / \mathrm{mL}$; procalcitonin, $0.72 \mathrm{ng} / \mathrm{mL}$; lactate dehydrogenase, $1531 \mathrm{U} / \mathrm{L}$; ferritin, $1251 \mathrm{ng} / \mathrm{mL}$; and C-reactive protein, greater than $27 \mathrm{mg} / \mathrm{L}$. Test results for Aspergillus, Blastomyces, Candida, Coccidioides and Histoplasma were negative. A new sample for SARS-CoV-2 IgG antibodies was sent, and the results of this test were again positive. Transthoracic echocardiography was performed, which showed a normal left ventricular ejection fraction and a small pericardial effusion. Although this was a limited evaluation, no coronary aneurysms at the ostial/proximal level were noted. 
This constellation of fever, tachycardia, hypoxia, GI tract symptoms, laboratory findings consistent with coagulopathy, elevated inflammatory markers with no evidence of other microbial cause of inflammation, and positive IgG antibodies for SARS-CoV-2 in a young patient is consistent with MIS-C. The cadiology service was consulted and agreed with this diagnosis. IVIG, $50 \mathrm{mg} / \mathrm{kg} / \mathrm{d}$, and oral aspirin, 20 to $25 \mathrm{mg} / \mathrm{kg}$ every 6 hours, were started. Her Kobayashi score was less than 5 , suggesting a low risk for nonresponsiveness to IVIG, so corticosteroids were not added to the regimen.

The patient had a dramatic improvement with treatment. Her low-grade fever and dyspnea resolved. In less than 48 hours, she was breathing without the need for supplemental oxygen. She was discharged home on high-dose aspirin with a tapering strategy (high-dose aspirin for 7 days and then $325 \mathrm{mg} / \mathrm{d}$ for 7 days), after which the plan was to reduce the dose to $81 \mathrm{mg} / \mathrm{d}$ thereafter until inflammatory marker levels and fevers improved. At follow-up a week after discharge, she was asymptomatic.

\section{DISCUSSION}

Epidemiologic data suggest that patients younger than 18 years who are infected with SARS-CoV-2 usually are asymptomatic or exhibit mild symptoms. In April 2020, European pediatric societies began alerting the medical community about a multisystemic inflammatory syndrome with features that overlap with conditions such as $\mathrm{KD}$ and, in more severe cases, toxic shock syndrome, occurring in previously healthy young patients with known exposure or who tested positive (via PCR or serologic assay) for SARS-CoV-2.5

A recent publication reported a 30 -fold increase in the incidence of KD and Kawasaki-like disease in the pediatric population in Italy's Bergamo province during $2020 .^{6}$ The city of Bergamo has the highest rate of SARS-CoV-2 infections and deaths in Italy. Later, several cases were also reported in the United States, specifically in New York City. ${ }^{7}$

Typical presentations consist of persistent fever and any of the following: hypotension, multiorgan compromise, and elevated inflammatory markers. Respiratory tract symptoms were not consistently present. ${ }^{5}$

In May 2020, the CDC published criteria for the diagnosis of MIS-C associated with COVID-19 (Table). ${ }^{4}$ According to the $\mathrm{CDC}$, all of the following must be present:

- An individual younger than 21 years presenting with fever, laboratory evidence of inflammation, and evidence of clinically severe illness requiring hospitalization, with multisystem $(\geq 2)$ organ involvement (cardiac, renal, respiratory, hematologic, gastrointestinal, dermatologic or neurologic)

- No alternative plausible diagnoses

- Positive for current or recent SARS-CoV-2 infection by RT-PCR, serology, or antigen test; or COVID-19 exposure within the 4 weeks prior to the onset of symptoms.

In this publication, the $\mathrm{CDC}$ also acknowledged that patients may also partially or fully meet the criteria for $\mathrm{KD}{ }^{4}$

The pathophysiology behind this multisystemic compromise can be explained by immune-mediated tissue damage secondary to the well-known COVID-19 cytokine storm resembling that of macrophage activation seen in viral-induced hemophagocytic lymphohistiocytosis. ${ }^{8-10}$

$\mathrm{KD}$ is a vasculitis of the medium to small-sized vessels with a predilection for the coronary arteries. It is usually self-limited and is seen mostly in children. ${ }^{11,12}$ Hemodynamic instability can be present initially, a condition known as $\mathrm{KD}$ shock syndrome. ${ }^{13}$ The physiopathology of this condition seems to be immunemediated and related to viral infection and macrophage activation syndrome. In some instances, adenovirus and coronavirus (ie, New Haven coronavirus) have been associated with KD, but the evidence for this association is not robust. ${ }^{14,15}$

No guidelines are available for the treatment of MIS-C associated with COVID-19. Case reports suggest good outcomes following treatment algorithms for KD: aspirin and IVIG (with corticosteroids if the Kobayashi score 5 or higher). ${ }^{16}$

Our patient met all the criteria for MIS-C associated with COVID-19: age, fever, inflammatory markers, multisystem compromise (hematologic, gastrointestinal, and possibly dermatologic), and she also met at least 2 criteria for $\mathrm{KD}$ (the rash was not clear and as such was not considered for diagnosis). She had 2 positive IgG antigen tests for SARS-CoV-2, and no alternative diagnoses were found. Empiric treatment with high-dose aspirin and IVIG led to dramatic clinical improvement of the patient's condition.

\section{SUMMARY}

As the COVID-19 pandemic unfolds, we continue to understand the clinical manifestations of this infection. It was initially assumed that the effects of this infection in the pediatric population were minimal. However, an increasing number of case reports of MIS-C associated with COVID-19/incomplete $\mathrm{KD}$ have emerged from various countries in Europe and Asia, and most recently from the United States.

The case presented here appears to be the first case reported in Indiana in an older individual (20 years) with rapid improvement after immunomodulating therapy (IVIG). The age of this patient is a reminder for all clinicians caring for COVID-19 patients to be aware of this emergent condition in young adults so as to allow early potentially life-saving therapy.

\section{REFERENCES:}

1. Lai C-C, Shih T-P, Ko W-C, Tang H-J, Hsueh P-R. Severe acute respiratory syndrome coronavirus 2 (SARS-CoV-2) and coronavirus disease-2019 (COVID-19): the epidemic and the challenges. Int J Antimicrob Agents. 2020;55(3):105924. doi:10.1016/j.ijantimicag.2020.105924

2. Zhu N, Zhang D, Wang W, et al. A novel coronavirus from patients with pneumonia in China, 2019. N Engl J Med. 2020;382(8):727-733. doi:10.1056/NEJMoa2001017

3. Ludvigsson JF. Systematic review of COVID-19 in children shows milder cases and a better prognosis than adults. Acta Paediatr. 2020;109(6):1088 1095. doi:10.1111/apa.15270

4. Centers for Disease Control and Prevention. Multisystem inflammatory syn- 
drome in children (MIS-C) associated with coronavirus disease 2019 (COVID-19). May 14, 2020. Accessed July 22, 2020. https://emergency.cdc. gov/han/2020/han00432.asp

5. Royal College of Paediatrics and Child Health. Guidance: paediatric multisystem inflammatory syndrome temporally associated with COVID-19. May 1, 2020. Accessed July 22, 2020. https://www.rcpch.ac.uk/sites/default/ files/2020-05/COVID-19-Paediatric-multisystem-\%20inflammatory\%20syndrome-20200501.pdf

6. Verdoni L, Mazza A, Gervasoni A, et al. An outbreak of severe Kawasaki-like disease at the Italian epicentre of the SARS-CoV-2 epidemic: an observational cohort study. Lancet. 2020;395(10239):1771-1778. doi:10.1016/S01406736(20)31103-X

7. New York City Health Department. 2020 Health alert \#13: pediatric multi-system inflammatory syndrome potentially associated with COVID-19. May 4, 2020. https://www1.nyc.gov/assets/doh/downloads/pdf/han/ alert/2020/covid-19-pediatric-multi-system-inflammatory-syndrome.pdf

8. Henderson LA, Canna SW, Schulert GS, et al. On the alert for cytokine storm: immunopathology in COVID-19. Arthritis Rheumatol. 2020;72(7): 1059-1063. doi:10.1002/art.41285

9. D'Antiga L. Coronaviruses and immunosuppressed patients: the facts during the third epidemic. Liver Transpl. 2020;26(6):832-834. doi:10.1002/ It.25756
10. Mehta P, McAuley DF, Brown M, et al; HLH Across Speciality Collaboration, UK. COVID-19: consider cytokine storm syndromes and immunosuppression. Lancet. 2020;395(10229):1033-1034. doi:10.1016/S0140-6736(20) 30628-0

11. Kawasaki T, Kosaki F, Okawa S, Shigematsu I, Yanagawa H. A new infantile acute febrile mucocutaneous lymph node syndrome (MLNS) prevailing in Japan. Pediatrics. 1974;54(3):271-276.

12. Kato $\mathrm{H}$, Sugimura T, Akagi T, et al. Long-term consequences of Kawasaki disease: a 10- to 21-year follow-up study of 594 patients. Circulation. 1996; 94(6):1379-1385. doi:10.1161/01.cir.94.6.1379

13. Kanegaye JT, Wilder MS, Molkara D, et al. Recognition of a Kawasaki disease shock syndrome. Pediatrics. 2009;123(5):e783-e789. doi:10.1542/ peds.2008-1871

14. Jaggi P, Kajon AE, Mejias A, Ramilo O, Leber A. Human adenovirus infection in Kawasaki disease: a confounding bystander? Clin Infect Dis. 2013;56(1): 58-64. doi:10.1093/cid/cis807

15. Esper F, Shapiro ED, Weibel C, Ferguson D, Landry ML, Kahn JS. Association between a novel human coronavirus and Kawasaki disease. J Infect Dis. 2005;191(4):499-502. doi:10.1086/428291

16. Sundel R. Kawasaki disease: initial treatment and prognosis. UpToDate. Updated June 26, 2020. Accessed July 22, 2020. https://www.uptodate.com/ contents/kawasaki-disease-initial-treatment-and-prognosis 